Received: 21.05 .2020

Revised: 30.06 .2020

Accepted: 24.07 .2020

DOI: $10.17804 / 2410-9908.2020 .4 .028-034$

\title{
DETERMINATION OF LITHIUM CONTENT IN SOLIDS BY NUCLEAR REACTION TECHNIQUE
}

\author{
V. B. Vykhodets ${ }^{1, \text { a) }}$, T. E. Kurennykh ${ }^{1, \text { b)* }}$, A. Yu. Nikolaev ${ }^{2,3, c)}$, \\ A. V. Suzdaltsev ${ }^{2,3, \text { d) }}$ and Yu. P. Zaikov ${ }^{2,3, e)}$ \\ ${ }^{1}$ M.N. Miheev Institute of Metal Physics, Ural Branch of the Russian Academy of Sciences, \\ 18, S. Kovalevskoy St., Ekaterinburg, 620137, Russian Federation \\ ${ }^{2}$ Institute of High-Temperature Electrochemistry, Ural Branch of the Russian Academy of Sciences, \\ 22, S. Kovalevskoy St. /20, Akademicheskaya St., Ekaterinburg, 620137, Russian Federation \\ ${ }^{3}$ B. N. Yeltsin Ural Federal University, \\ 19, Mira St., Ekaterinburg, 620002, Russian Federation \\ a) iD http://orcid.org/0000-0001-9522-9147; \\ b) (iD http://orcid.org/0000-0001-9859-3374; \\ c) iD http://orcid.org/0000-0001-5931-8812; \\ d) (iD http://orcid.org/0000-0003-3004-7611; \\ e) (iD http://orcid.org/0000-0001-6138-3955
}

*Corresponding author: E-mail: kurennykh@imp.uran.ru

Address for correspondence: 18, S. ul. Kovalevskoy, Ekaterinburg, 620990, Ekaterinburg, Russian Federation Tel.: +7 (343) 3783846

The acceleration method of nuclear reactions is used to measure the content of lithium and oxygen in zirconium oxides. The purpose of the study stems from the fact that there are no direct methods for determining lithium concentration in solids, while lithium is currently widely used for alloying alloys in aircraft construction, nuclear power engineering, electrochemical devices, and other fields of technology. It is shown that satisfactory metrological characteristics of the method are provided when using the ${ }^{6} \mathrm{Li}\left(\mathrm{d}, \mathrm{p}_{0}\right)^{7} \mathrm{Li}$ and ${ }^{7} \mathrm{Li}(\mathrm{d}, \mathrm{p})^{8} \mathrm{Li}$ reactions at a deuteron energy of $650 \mathrm{keV}$.

Keywords: lithium, zirconium oxides, nuclear microanalysis, electrolysis, reduction.

\section{Acknowledgment}

The research was carried out within the state assignment from the Ministry of Science and Higher Education of the Russian Federation (theme Function, No. AAAA-A19-119012990095-0).

\section{References}

1. Elwyn A.J., Holland R.E., Davids C.N., Meyer-Schutzmeister L., Monahan J.E., Mooring F.P., Ray W. Absolute cross sections for deuteron-induced reactions on Li at energies below $1 \mathrm{Mev}$. Physical Review C, 1977, vol. 16, no. 5, pp. 1744-1756. DOI: 10.1103/PhysRevC.16.1744.

2. Ion Beam Handbook for Material Analysis, ed. by J.W. Mayer and E. Rimini, Academic Press, 1977, 488 p. ISBN 978-0-12-480860-7.

3. Choi Eu.Y., Lee J. Complete reduction of high-density $\mathrm{UO}_{2}$ to metallic $\mathrm{U}$ in molten $\mathrm{Li}_{2} \mathrm{O}-\mathrm{LiCl}$. Journal of Nuclear Materials, 2017, vol. 494, pp. 439-447. DOI: 10.1016/j.jnucmat.2017.07.036.

4. Mullabaev A., Tkacheva O., Shishkin V., Kovrov V., Zaikov Y., Sukhanov L., Mochalov Y. Properties of the $\mathrm{LiCl}-\mathrm{KCl}-\mathrm{Li}_{2} \mathrm{O}$ system as operating medium for pyro-chemical reprocessing of spent nuclear fuel. J. Nuclear Materials, 2018, vol. 500, pp. 235-241. DOI: 10.1016/j.jnucmat.2018.01.004. 
5. Sakamura Y., lizuka M., Kitawaki S., Nakayoshi A., Kofuji H. Formation and reduction behaviors of zirconium oxide compounds in $\mathrm{LiCl}-\mathrm{Li}_{2} \mathrm{O}$ melt at $923 \mathrm{~K}$. J. Nuclear Materials, 2015, vol. 66, pp. 269-279. DOI: 10.1016/j.jnucmat.2015.08.011.

6. Choi Eu.Y., Heo D.H. Reduction of zirconium oxide compounds by lithium metal as a reductant in molten LiCl salt. J. Nuclear Materials, 2018, vol. 512, pp. 193-198. DOI: 10.1016/j.jnucmat.2018.10.015.

7. Baraboshkin A.N. Elektrokristallizatsiya metallov iz rasplavlennykh solei [Electrocrystallization of Metals from Molten Salts]. Moscow, Nauka Publ., 1976, 280 p. (In Russian).

8. The Stopping and Ranges of Ions in Matter-SRIM2013. Available at: http://www.srim.org 
Подана в журнал: 21.05.2020

УДК 544.653.3

DOI: $10.17804 / 2410-9908.2020 .4 .028-034$

\title{
ОПРЕДЕЛЕНИЕ СОДЕРЖАНИЯ ЛИТИЯ В ТВЕРДЫХ ТЕЛАХ МЕТОДОМ ЯДЕРНЫХ РЕАКЦИЙ
}

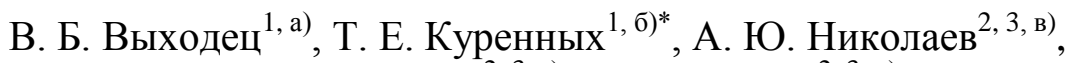 \\ А. В. Суздальцев ${ }^{2,3, \text { г) }}$, Ю. П. Зайков ${ }^{2,3, \text { д) }}$ \\ ${ }^{1}$ Федеральное государственное бюджетное учреждение науки Институт физики металлов им. М.Н. Михеева \\ Уральского отделения Российской академии наук, \\ 18, ул. С. Ковалевской, Екатеринбург, Российская Федерация \\ ${ }^{2}$ Федеральное государственное бюджетное учреждение науки \\ Институт высокотемпературной электрохимии Уральского отделения Российской академии наук, \\ 20, ул. Академическая, Екатеринбург, Российская Федераџия \\ ${ }^{3}$ Уральский федеральный университет имени первого Президента России Б.Н. Ельцинн, \\ 19, ул. Мира, Екатеринбург, Российская Федерация \\ a) (iD http://orcid.org/0000-0001-9522-9147; \\ б) (D) http://orcid.org/0000-0001-9859-3374; \\ в) (iD http://orcid.org/0000-0001-5931-8812; \\ r) (DD http://orcid.org/0000-0003-3004-7611; \\ य) (iD http://orcid.org/0000-0001-6138-3955
}

\begin{abstract}
*Ответственный автор. Электронная почта: kurennykh@imp.uran.ru Адрес для переписки: ул. С. Ковалевской, 18, Екатеринбург, Российская Федерация
\end{abstract} Тел.: +7 (343) 378-38-46

Ускорительная методика ядерных реакций применена для измерения содержания лития и кислорода в оксидах циркония. Постановка исследования обусловлена тем, что для лития отсутствуют прямые методы определения его концентрации в твердых телах, а в настоящее время литий широко применяется для легирования сплавов в авиастроении, ядерной энергетике, электрохимических устройствах и других областях техники. Удовлетворительные метрологические характеристики методики обеспечиваются при использовании реакций ${ }^{6} \mathrm{Li}\left(\mathrm{d}, \mathrm{p}_{0}\right){ }^{7} \mathrm{Li} \mathrm{и}{ }^{7} \mathrm{Li}(\mathrm{d}, \mathrm{p}){ }^{8} \mathrm{Li}$ при энергии дейтронов 650 кэВ.

Ключевые слова: литий, оксиды циркония, ядерный микроанализ, электролиз, восстановление.

\section{1. Введение}

Литий и его соединения находят широкое применение в различных областях техники. $\mathrm{B}$ частности, в ядерной энергетике изотоп ${ }^{6} \mathrm{Li}$ служит промышленным источником для производства трития, а изотоп ${ }^{7} \mathrm{Li}$ используется как теплоноситель. В настоящее время литийионные аккумуляторы являются основным источником питания для всех автономных электрических устройств, включая смартфоны, электромобили и межпланетные зонды. Легирование литием придает уникальные свойства алюминиевым сплавам: повышает их прочность, модуль упругости, уменьшает плотность, многие литий-алюминиевые сплавы можно сваривать в отличие от алюминиевых. Сплавы с литием находят все более широкое распространение в авиационном машиностроении. В связи с этими и другими применениями лития актуальной является задача определения концентрации лития в металлических сплавах и оксидах. Цель работы - применение ускорительной методики ядерных реакций (NRA) для измерения концентрации лития в твердых телах и оценка метрологических характеристик методики NRA в этом случае. Постановка исследования обусловлена тем, что существующие 
спектральные методы не обеспечивают получение надежных результатов для изотопов легких элементов, в том числе для лития, а методика NRA успешно применялась для многих изотопов легких элементов, например, ${ }^{2} \mathrm{H},{ }^{16} \mathrm{O},{ }^{18} \mathrm{O},{ }^{14} \mathrm{~N},{ }^{15} \mathrm{~N},{ }^{12} \mathrm{C}$. Для измерения концентрации изотопов лития в твердых телах методика NRA практически не применялась, в то же время при облучении ускоренными дейтронами и протонами на изотопах лития протекают ядерные реакции ${ }^{6} \mathrm{Li}\left(\mathrm{d}, \mathrm{p}_{0}\right)^{7} \mathrm{Li}{ }^{7} \mathrm{Li}(\mathrm{d}, \mathrm{p})^{8} \mathrm{Li}$ с большим выходом протонов $[1,2]$.

\section{2. Образцы и методика}

Апробация методики NRA для изотопов лития проводилась при исследовании процесса восстановления оксида $\mathrm{ZrO}_{2}$ при электролизе расплава $\mathrm{LiCl}-\mathrm{KCl}-\left(1\right.$ мас. \%) $\mathrm{Li}_{2} \mathrm{O}$. Постановка такого исследования обусловлена тем, что расплавленные смеси на основе хлорида лития являются перспективными средами для осуществления ряда операций пирохимической переработки отработавшего ядерного топлива $[3,4]$. Одной из таких операций является восстановление окисленного топлива при электролизе расплавов $\mathrm{LiCl}-\mathrm{Li}_{2} \mathrm{O}$ или LiCl$\mathrm{KCl}-\mathrm{Li}_{2} \mathrm{O}$. Сущность операции заключается в том, что выделяющийся при электролизе указанных расплавов литий селективно восстанавливает оксиды актинидов до соответствующих металлов, которые используют для изготовления топлива. В составе отработанного ядерного топлива в значительном количестве присутствует $\mathrm{ZrO}_{2}$, данные о степени восстановления литием которого противоречивы $[5,6]$.

Для приготовления использовали индивидуальные хлориды $\mathrm{LiCl}$ и $\mathrm{KCl}$, которые поэтапно нагревали под вакуумом, переплавляли в аргоне, после чего подвергали зонной перекристаллизации [7]. Оксид в готовый расплав добавляли в виде предварительно приготовленного концентрата $\mathrm{LiCl}-\mathrm{Li}_{2} \mathrm{O}$ [4]. Все операции, включая хранение солей, выполнение экспериментов и вспомогательные операции проводили в сухом аргоновом боксе.

Электрохимические измерения и электролиз проводили в стальном контейнере с исследуемым расплавом, который дополнительно изолировали от атмосферы бокса в кварцевой пробирке с фторопластовой крышкой. В крышке размещали противоэлектрод, два рабочих молибденовых электрода с одинаковой геометрической поверхностью (эталонный Мо и погруженный в тигель $\mathrm{MgO}$ с порошком $\mathrm{ZrO}_{2}$ электрод $\mathrm{Mo} / \mathrm{ZrO}_{2}$ ), термопару и квазиэлектрод сравнения Bi-Li. Для проведения измерений и электролиза использовали PGSTAT AutoLab 320N с ПО NOVA 1.11 (TheMetrOhm, Нидерланды). Температуру расплава измеряли и поддерживали при помощи термопары $\mathrm{Pt} / \mathrm{PtRh}$ и модуля USB-TC01 (NationalInstruments, CША). Электролиз вели при температуре расплава $650{ }^{\circ} \mathrm{C}$ в импульсном гальваностатическом режиме, обеспечивающем выделение лития и фиксацию потенциала электрода при отключении тока.

По окончании электролиза восстановленный порошок $\mathrm{ZrO}_{2}$ извлекали из тигля $\mathrm{MgO}$ и промывали в дистиллированном спирте. Для определения состава восстановленного порошка использовали рентгенофазовый (XRD) анализ, метод карботермического восстановления и NRA. В последнем случае для определения содержания кислорода и лития в исследуемом порошке восстановленного оксида циркония использовали реакции ${ }^{16} \mathrm{O}\left(\mathrm{d}, \mathrm{p}_{0}\right){ }^{17} \mathrm{O},{ }^{6} \mathrm{Li}\left(\mathrm{d}, \mathrm{p}_{0}\right)^{7} \mathrm{Li}$ и ${ }^{7} \mathrm{Li}(\mathrm{d}, \mathrm{p})^{8} \mathrm{Li}$. Источником ускоренных дейтронов служил $2 \mathrm{MB}$ ускоритель Ван де Граафа, энергия частиц первичного пучка дейтронов 650 кэВ. Спектры ядерных реакций регистриро-

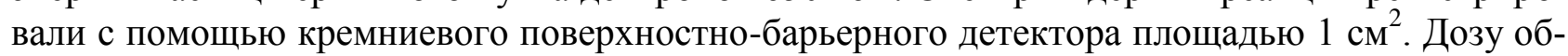
лучения образцов измеряли с помощью вторичного монитора с погрешностью около $1,5 \%$. Измерения методом ядерных реакций выполнены на порошках. Для этого частицы порошка впрессовывали в пластину индия. В результате вблизи наружной поверхности образца формировался слой толщиной не менее 2 мкм, содержащий только частицы оксида. Плоская поверхность образцов устанавливалась перпендикулярно оси первичного пучка.

Для вычисления значений концентраций кислорода и лития использовалась процедура сравнения спектров от исследуемого образца и образцов-эталонов с постоянной по глубине 
концентрацией кислорода и лития. В качестве эталонов использовался порошок $\mathrm{ZrO}_{2}$, содержание кислорода в котором в соответствии со стехиометрической формулой принималось равным 67 ат. \% (26 мас. \%), и $\mathrm{Li}_{2} \mathrm{ZrO}_{3}$, содержание лития в котором в соответствии со стехиометрической формулой принималось равным 33,33 ат. \% (9,06 мас. \%). Значения концентраций вычислялись с использованием табулированных данных по тормозным способностям элементов [8].

\section{3. Результаты и обсуждение}

Вольтамперные характеристики электролиза свидетельствовали о протекании в различных диапазонах напряжений и временных интервалов как процессов выделения лития на катоде, так и расходования восстановленного лития на последующие химические реакции, например, на восстановление порошка $\mathrm{ZrO}_{2}$.После электролизных испытаний порошок $\mathrm{ZrO}_{2}$ приобрел темно-серую окраску. Согласно данным XRD анализа порошок после отмывки от солей содержал фазы $\mathrm{ZrO}_{2}, \mathrm{Li}_{2} \mathrm{ZrO}_{3}$ и $\mathrm{ZrO}_{0,334}$. По данным метода карботермического плавления в исходном порошке $\mathrm{ZrO}_{2}$ содержалось $(26,3 \pm 0,95)$ мас. \% кислорода, после восстановления наблюдалось понижение его содержания до $(22,7 \pm 1,5)$ мас. \%.

На рисунке представлены спектры продуктов ядерных реакций.

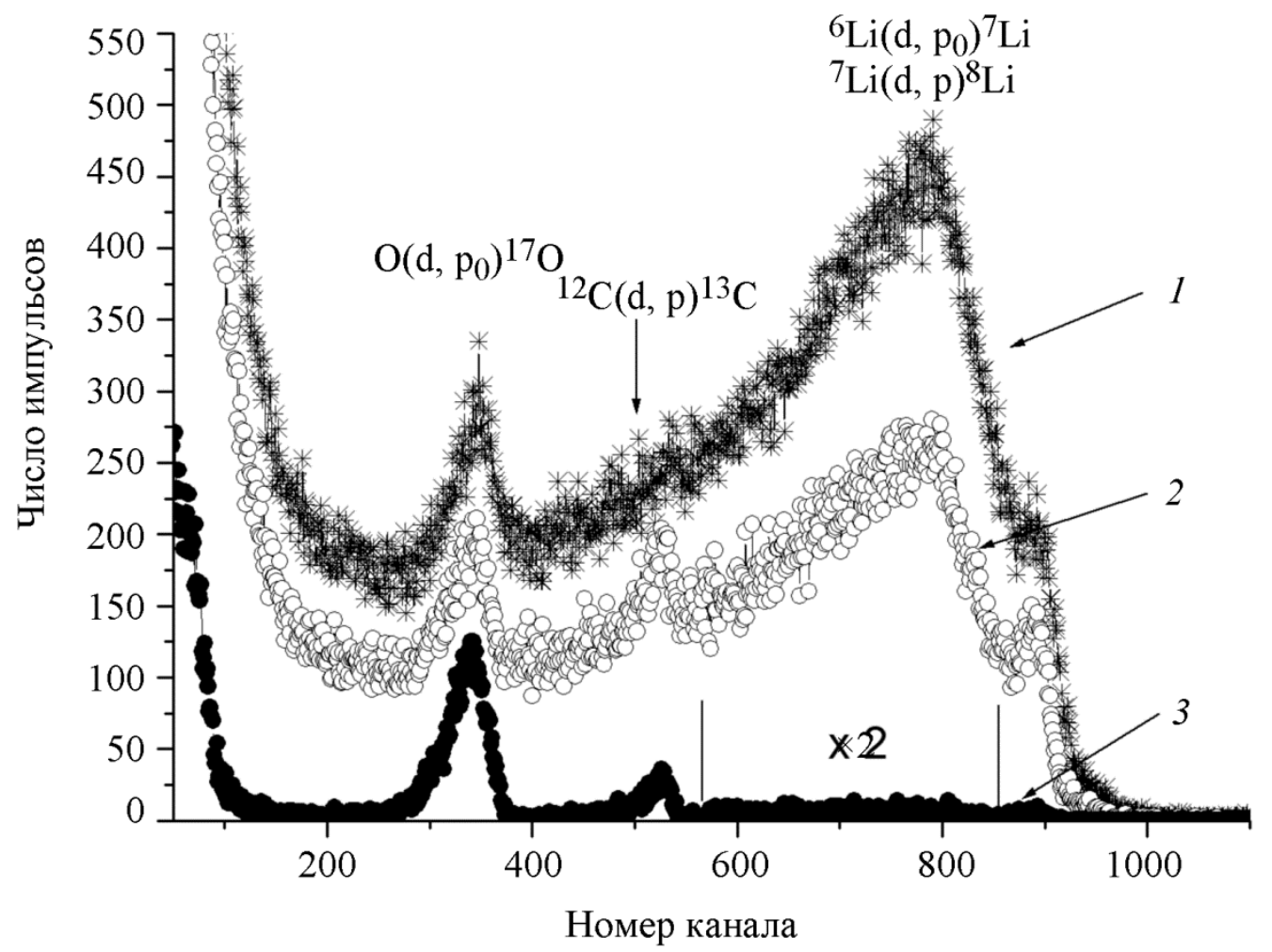

Спектры ядерных реакций от исследуемого порошка и эталонов: $1-\mathrm{Li}_{2} \mathrm{ZrO}_{3} ; 2$ - восстановленный $\mathrm{ZrO}_{2} ; 3-\mathrm{ZrO}_{2}$

Из спектра для восстановленного оксида циркония видно, что порошок содержит в значительном количестве литий. Спектры лития накладываются на спектр кислорода, и это осложняло определение концентрации кислорода в порошке. По этой причине для определения концентрации кислорода в порошке использовали процедуру вычитания спектров. Она, конечно, приводила к значительной ошибке в определении концентрации кислорода. Были сделаны попытки найти более корректную процедуру для определения концентрации кислорода, для этого проводили дополнительные эксперименты с облучением образцов не дейтронами, а протонами, измеряли концентрацию не только изотопа ${ }^{16} \mathrm{O}$, но и ${ }^{18} \mathrm{O}$, регистрировали 
продукты реакций от изотопов лития ${ }^{6} \mathrm{Li}$ и ${ }^{7} \mathrm{Li}$, варьировали энергии дейтронов и протонов, но улучшить ситуацию не удалось.

Таким образом, можно констатировать, что изотопы лития являются благоприятными объектами для определения их концентрации в твердых телах с помощью методики NRA. $\mathrm{C}$ ее помощью концентрации лития на уровне 0,001 ат. \% и выше могут быть измерены с точностью несколько процентов. Такой уровень измерений характерен в методике NRA для изотопов с высокими сечениями ядерных реакций. В то же время присутствие лития в образцах в значительных количествах будет осложнять измерение с помощью методики NRA низких концентраций изотопов других легких элементов, например кислорода, азота и углерода.

При обработке представленных на рисунке спектров было установлено, что восстановленный при электролизе порошок имеет следующий состав: $\mathrm{ZrLi}_{0,87} \mathrm{O}_{1,98}$. Погрешности в индексах лития и кислорода были на уровне $2-3 \%$. Из формульной записи состава оксида легко получить, что массовая доля кислорода в исследуемом порошке составила $(24,6 \pm 1)$ мac. \%, Это значение, установленное с помощью методики NRA, в пределах статистической погрешности методик согласуется с таковым, полученным методом карботермического плавления: $(22,7 \pm 1,5)$ мас. \%. Методики ядерных реакций и карботермического плавления очень сильно отличаются по принципам, и такое согласие, конечно, свидетельствует о надежности обеих методик. Выше отмечалось, что по данным XRD анализа восстановленный порошок содержал фазы $\mathrm{ZrO}_{2}, \mathrm{Li}_{2} \mathrm{ZrO}_{3}$ и $\mathrm{ZrO}_{0,334}$. При использовании этих результатов и формульной записи оксида убеждаемся, что на 1 моль оксида $\mathrm{ZrO}_{2}$ в восстановленном порошке приходится 0,67 моля цирконата лития $\mathrm{Li}_{2} \mathrm{ZrO}_{3}$ и 0,41 моля оксида $\mathrm{ZrO}_{0,334}$. Таким образом, полученные в работе данные свидетельствуют о том, что при электролизе с высокой эффективностью происходило восстановление $\mathrm{ZrO}_{2}$ преимущественно до оксидов циркония низшей валентности и цирконатов лития. Восстановления до металлического циркония в работе не обнаружено.

\section{4. Заключение}

В работе показано, что применение ускорительной методики ядерных реакций позволяет определять концентрации лития в твердых телах выше 0,001 ат. \% с точностью до нескольких процентов от измеряемой величины. Этот уровень измерений достаточен для решения большинства задач, возникающих при использовании лития в авиастроении и других областях техники.

Получены новые данные о восстановлении диоксида циркония $\mathrm{ZrO}_{2}$ при электролизе расплавов на основе хлорида лития. Установлено, что восстановление происходит преимущественно до оксидов циркония низшей валентности и цирконатов лития, при этом степень восстановления до металлического циркония крайне мала.

\section{Благодарность}

Работа выполнена в рамках государственного задания МИНОБРНАУКИ России (тема «Функиия», № AAAA-A19-119012990095-0).

\section{Литература}

1. Absolute cross sections for deuteron-induced reactions on Li at energies below $1 \mathrm{Mev} /$ A. J. Elwyn, R. E. Holland, C. N. Davids, L. Meyer-Schutzmeister, J. E. Monahan, F. P. Mooring, W. Ray // Physical Review C. - 1977. - Vol. 16, no. 5. - P. 1744-1756. DOI: 10.1103/PhysRevC.16.1744.

2. Ion Beam Handbook for Material Analysis / ed. by J. W. Mayer and E. Rimini. - Academic Press, 1977. - 488 p. - ISBN 978-0-12-480860-7.

3. Choi Eu. Y., Lee J. Complete reduction of high-density $\mathrm{UO}_{2}$ to metallic $\mathrm{U}$ in molten $\mathrm{Li}_{2} \mathrm{O}-\mathrm{LiCl} / /$ Journal of Nuclear Materials. - 2017. - Vol. 494. - P. 439-47. - DOI: 10.1016/j.jnucmat.2017.07.036. 
4. Properties of the $\mathrm{LiCl}-\mathrm{KCl}-\mathrm{Li}_{2} \mathrm{O}$ system as operating medium for pyro-chemical reprocessing of spent nuclear fuel / A. Mullabaev, O. Tkacheva, V. Shishkin, V. Kovrov, Y. Zaikov, L. Sukhanov, Y. Mochalov // J. Nuclear Materials. - 2018. - Vol. 500. - P. 235-241. DOI: 10.1016/j.jnucmat.2018.01.004.

5. Formation and reduction behaviors of zirconium oxide compounds in $\mathrm{LiCl}-\mathrm{Li}_{2} \mathrm{O}$ melt at 923 K / Y. Sakamura, M. Iizuka, S. Kitawaki, A. Nakayoshi, H. Kofuji // J. Nuclear Materials. 2015. - Vol. 66. - P. 269-279. - DOI: 10.1016/j.jnucmat.2015.08.011.

6. Choi Eu. Y., Heo D. H. Reduction of zirconium oxide compounds by lithium metal as a reductant in molten $\mathrm{LiCl}$ salt // J. Nuclear Materials. - 2018. - Vol. 512. - P. 193-198. DOI: 10.1016/j.jnucmat.2018.10.015.

7. Барабошкин А. Н. Электрокристаллизация металлов из расплавленных солей. М. : Наука, 1976. - 280 c.

8. The Stopping and Ranges of Ions in Matter-SRIM2013. - URL: http://www.srim.org 